\title{
Les contes facétieux et anecdotes avec trickster au Portugal
}

\author{
Paulo Correia \\ Universidade do Algarve \\ paulojcorreia66@gmail.com
}

\begin{abstract}
RÉSUMÉ
Mon apport se fera autour d'une réflexion sur un corpus de récits brefs regroupés (quand il est possible les cataloguer) sur les catégories «Tales of the stupid ogre» (ATU IOoO-II99) et «Anecdotes and Jokes» (ATU I200-I999). Le propos c'est de vérifier comment, dans cet encadrement de forme (court et en épisodes) et de caractère (facétieux, voire critique), le personnage du héros malin est paradigme du petit peuple. Les personnages de Bocage (poète portugais du XVIII siècle) et Pedro das Malazartes (lit. «Pierre des Maux-Arts») sont le sommet de ce genre de héros, qui parfois a des allures de héros «national», malgré son existence partout en Péninsule Ibérique et ainsi sur l'Amérique du sud. Néanmoins, il y a un autre genre de héros, plus attaché à une géographie précise-le personnage de l'alentejano- paresseux, villageois, mais malin et qui donne des leçons aux gens de la grande ville. Tandis que le premier type de héros apparaît sur des facéties classifiables d'un numéro ATU, le deuxième est surtout sujet des histoires drôles, plaisanteries, auxquelles on ne peut pas attribuer un numéro ATU. C'est autour de ces deux groupes d'histoires courtes qu'on fera une réflexion sur les limites floues des genres courts facétieux oraux.
\end{abstract}

MOTS CLÉS

conte facétieux, anecdote, ATU, héros, genre court

\section{RESUM}

La meva aportació es farà al voltant d'una reflexió sobre un corpus de relats breus agrupats (quan és possible catalogar-los) en les categories «Tales of the stupid ogre» (ATU Iooo-II99) $i$ «Anecdotes and Jokes» (ATU I20o-I999). L'objectiu és comprovar com, en aquest enquadrament formal (curt i en episodis) i de caràcter (graciós, fins i tot crític), el personatge de l'heroi malvat és paradigma de la gent del poble. Els personatges de Bocage (poeta portuguès del segle XVIII) i Pedro das Malazartes (lit. «Pere de les Males Arts») són l'exemple màxim d'aquest gènere d'heroi, que de vegades s'assembla a un heroi «nacional», malgrat la seva existència arreu de la península Ibèrica i també a l'Amèrica del Sud. Tanmateix, hi ha un altre tipus d'heroi, més lligat a una geografia precisa-el per- 


\title{
Paulo Correia
}

sonatge de l'alentejano- mandrós, pobletà, però astut i que dóna lliçons a la gent de la ciutat. Mentre que el primer tipus d'heroi apareix en acudits classificables en un número ATU, el segon és, sobretot, protagonista d'històries divertides, d'acudits, als quals no es pot pas atribuir un número ATU. És al voltant d'aquests dos grups de relats curts que es farà una reflexió sobre els límits difusos dels gèneres curts graciosos orals.

PARAULES CLAU

facècia, anècdota, ATU, heroi, gènere curt

\begin{abstract}
My contribution will be a reflection on a corpus of short stories grouped (whenever it is possible to catalogue them) into the categories of the "Tales of the stupid ogre" (ATU IOOO - II99) and the "Anecdotes and Jokes" (ATU I2OO - I999). The aim is to determine to what extent, within these short, episodic, funny and even critical forms, the character of the trickster is a paradigm of village folk. The characters of Bocage (I8th century Portuguese poet) and Pedro das Malazartes (lit. Peter of the Evil Arts) are examples of this genre of hero, a figure that has at times adopted the status almost of "national hero of Portugal", despite the fact that he can be found throughout the Iberian Peninsula and South America. However, there is another type of hero who is more closely tied to a geographical place: the alentejà. He is lazy and bucolic but also astute and able to outwit the townsfolk. Whereas the first type of hero appears in jokes that can be assigned an ATU number, the second is above all the protagonist of funny stories or jokes which cannot classified according to the ATU. I will therefore use these two groups to reflect on the imprecise limits of short, oral anecdotes and jokes.
\end{abstract}

\section{KEYWORDS}

joke, anecdote, ATU, hero, short form 
LA FIGURE MYTHIQUE DU «TRICKSTER » est bien connue par des chercheurs en littérature orale. Cet archétype apparaît sur les récits oraux traditionnels de peuples très diversifiés et écartés géographiquement. On peut mentionner, à titre d'exemple, l'œuvre de Paul Radin sur les récits de trickster des indiens Winnebago et Tlingit de l'Amérique du Nord (Radin I972), ou celle de Edward Evans-Pritchard sur l'ethnie Azande, en Afrique Noire (Street 1972), et encore, les études de Marcel Griaule sur la figure du « Renard Pâle » des Dogon (Griaule-Dieterlen I965). En faisant son apparition surtout comme un animal (corbeau, renard, araignée, etc.) sur des narratives de teneur mythique, ce héros est quelqu'un porteur d'un caractère double, simultanément générateur de désordre, mais aussi donneur de culture aux hommes. Est surtout un être liminal, dont le comportement se fait dans le seuil des mœurs de la société humaine. Ce demi-dieu civilisateur reste, malgré tout, dans la sphère des narrations sacrées des peuples appelés «primitifs ».

Un autre avatar de cette créature « à mille visages » est Djohá, connu autour le bassin de la Méditerranée, au moins dés le $\mathrm{X}^{\mathrm{e}}$ siècle, surtout parmi les peuples arabes et juifs. Ces récits facétieux, circulaient non seulement bouche à oreille mais aussi sur bouquins de colportage. Cette littérature drolatique a pour matière les aventures d'un héros niais qui montre aussi de ruse que de naïveté dans ses actions, et que, jusqu'à aujourd'hui provoquent des sourires simplement en disant son nom. On peut rencontrer ses histoires en Sicile (où il est nommé Giufà), et un peu partout au Moyen-Orient et Nord d'Afrique.

Mais notre sujet ici c'est l'avatar Ibérique de ce tricheur. Aussi très connu, il peut prendre des identités diversifiées. Dans la Péninsule Ibérique on le connaît par le nom de Pedro Malasartes (au Portugal) et Pedro Urdemalas (en Espagne) (fig. I). Le nom « de famille » du héros, qui a plusieurs variations régionales, montre bien son caractère générateur de désordre: en traduction littérale en français ça signifie Pierre des Mauvaises Arts. Il est connu non seulement sur l'Europe, mais aussi partout en Amérique Latine, apporté par les émigrants espagnols et portugais le long des siècles de colonisation du nouveau monde. Il est l'archétype du « malandro » mot que veut dire simultanément paresseux et malin. Son nom et ses histoires son très, très connues partout où on parle espagnol et portugais.

Un autre trait de ce personnage c'est qu'il adopte des noms d'écrivains célèbres. En Espagne, Quevedo est peut-être le plus connu. Au Portugal, nous avons son homologue: Bogage, un poète du XVIII ${ }^{\mathrm{e}}$ siècle et auteur de poésies satiriques et érotiques (fig. 2). Ces deux personnages apparaissent surtout dans des anecdotes très courtes de teneur parfois eschatologique ou érotique.

Au Brésil, ce personnage est interchangeable avec un autre dont le nom, Camões, est celui d'un poète portugais $\mathrm{du} \mathrm{XVI}^{\mathrm{e}}$ siècle, auteur des «Lusiades» notre 
épopée nationale (fig. 3). Les deux ont mené une vie d'aventures, selon la légende, et c'est peut-être là la raison du choix de leurs noms pour ces personnages de transgression.

Tout de suite, nous allons voir sur quels genres de récits oraux se promènent ces personnages.

Tout d'abord, c'est sur la catégorie des Contes du Géant Dupé(ATU Iooo-II99), qu'on va trouver Pedro Malasartes en confrontation avec un mauvais patron ou un géant. D'une part, il va se profiter d'un contrat de travail déshonnête pour dilapider le patrimoine de son patron tout en faisant, au pied de la lettre, les taches impossibles que celui-là l'impose. D'autre part, en compétition avec un géant, il triche de façon à se profiter de la force de son opposant. Dans les deux cas, le héros, grâce à la ruse, est toujours le vainqueur sur un opposant plus puissant que lui-même, en rang social ou en force physique. On commence déjà à voir que ce genre de récits peut illustrer une sorte de "lutte de classes » ou notre tricheur est en représentation du petit peuple. Un anthropologue brésilien, Roberto Da Matta, fait une lecture " sociologique » de ces narratives en épisodes multiples sous l'égide du contrat de travail, qui va dans ce sens (Da Matta I983: 193-235).

On est, donc, tombé sur des récits courts enchainés en narrations plus ou moins longs où le personnage central est Pedro Malasartes. Comment est-ce que ces narrations circulent dans l'espace lusophone? Selon les informations disponibles, ces histoires sont bien représentées au Portugal et encore mieux au Brésil (voir Rodriguez I985). Hors de ces deux pays, on va les trouver seulement dans les îles de Cap-Vert avec un héros avec un nom différent.

C'est de noter que dans la partie ou l'opposant extraordinaire est un diable (ATU II45-II99), le héros n'est plus Pedro Malasartes. Néanmoins, il réapparait au long des contes facétieux (ATU I200-I999).

Sur un autre environnement, son caractère change un peu, et Malasartes peut prendre la place du niais qui vit encore avec sa mère, qu'il aide d'une façon maladroite: à la maison, pour faire écluser les oufs de la poule (ATU I68IB), ou bien hors de chez soi, en faisant ce que sa mère le demande (ATU I696: Qu'est ce que je devrais faire/dire?), parfois avec des coups de chance, en faisant fuir des voleurs et prenant leur argent (ATU I653: Les voleurs sur l'arbre).

D'autres fois il s'en mêle dans les affaires des autres, notamment sur les amours secrètes d'une femme adultère, en gagnant toujours argent ou nourriture, en profit propre (ATU I358A) ou du mari légitime (ATU I358C). Un fait sûr, c'est qu'il met tous ses ruses au service de ses appétits primaires, soit de nourriture (ATU I74I : Les oreilles du curé; ATU I54I: Pour le Mai), de sexe (ATU I563: les deux?), ou d'argent.

Mas ses tricheries peuvent aussi se manifester par actions de pur vol (ATU I525D et ATU I525E) ou bien, par des méfaits pour épater son patron, une fois de plus (ATU I33ID* et ATU I562A).

Il est de mentionner aussi le conte-type ATU I535 (Le paysan riche et le pauvre), ou Pedro Malasartes (ou Camões) surgit comme protagoniste seulement au Brésil. $\mathrm{Au}$ Portugal, il y a beaucoup de versions connues de ce récit, mais leur protagoniste est Jean le Sot. Cet exemple est un de plusieurs que montre bien la profusion des avatars secondaires de la figure archétypale du trickster collés à des antihéros niais et malins à la fois.

Bocage met aussi en œuvre ses tricheries pour se profiter des autres et satisfaire ses besoins primaires de nourriture (comme étudiant, il triche pour s'en sortir 
sans payer son repas: ATU I526A), et sexe (en séduisant les filles de son patron : ATU I563). La différence entre Malasartes et Bocage c'est que le dernier affronte des sujets avec un dégrée plus fort de transgression des tabous, soit la mort (ATU I537: Le cadavre mort plusieurs fois), le mépris de l'autorité (Hansen ${ }^{* *}$ I552: Appeler pute à la reine et cocu au roi), et surtout les thèmes eschatologiques (ATU I38IB : Le jour dans lequel la merde a tombé du ciel; et Hansen ** I709 C: Bocage est reconnu par des femmes à cause de son cul). Ce dernier récit est une plaisanterie minimale, connue en Espagne dans une anecdote de Quevedo.

Hors des facéties, il faut dire que le personnage de Bocage apparaît aussi dans les contes-nouvelles : dans le ATU $900 C^{*}$, ou il fait semblé d'être muet pour séduire des jeunes filles, et dans le ATU 924, où il profite d'une discussion en signes pour faire des gestes obscènes.

De tous ces contes-type très différents, nous allons nos pencher un peu plus en détail sur le ATU I528 (Tenant le chapeau par terre). La raison de ce choix c'est parce qu'il s'agit d'une histoire court, pose le problème de distinction entre conte facétieux et plaisanterie (voire anecdote), et surtout parce qu'on y va trouver, dans les versions lusophones de ce type, les trois tricheurs dont nous parlons: Pedro Malasartes, Bocage et Camões.

Pour prendre l'histoire de son vif, voilà une version recueillie à Vila Marim, village du département de Vila Real, province des Trás-os-Montes, au Nord du Portugal. L'informatrice est Maria Celeste Macedo, de 68 ans.

Un jour, Bocage marchait le long d'un chemin, et de loin, s'aperçoit venir deux personnes bien habillées, riches. Alors qu'est ce qu'il a fait? Il a plié ses jambes, fait caca et mit son chapeau par terre pour la couvrir.

Entretemps, les autres s'approchaient et lui ont demandé:

-Bocage, qu'est que tu fais là?

-Je suis là parce que j'ai attrapé un oiseau. J'avais besoin d'aller acheter une cage, mais je ne peux pas, car j'ai mis ici mon chapeau et j'avais besoin d'un pour y aller.

Volontiers, ils lui donnent un chapeau et il fut tout de suite acheter une cage, en les laissant là pour garder l'oiseau. Et ils n'ont pas quitté la main du chapeau.

Pedro ne revenait pas. Ils s'ennuyaient d'avoir sa main sur le chapeau et on pensé jeter un coup d'œil en dessous du chapeau, pour apporter l'oiseau chez soi. Ils on mit la main et on attrapé de la merde! ${ }^{\text {I }}$

Il y a, dans les archives portugaises du conte, 5 versions de ce type, et, à l'exception de la version présentée, tous les autres ont été enregistrés au Canada et en Californie, de la bouche des portugais émigrants nés dans les îles des Azores et Madère. Dans l'ensemble, on est en présence des trois régions portugaises plus isolées (Azores, Madère et Trás-os-Montes), qui sont simultanément les plus archaïsantes en matière de tradition orale. Cela peut-être un signe de l'ancienneté de cette histoire, ce qui l'éloigne de la simple plaisanterie contemporaine. An autre signe d'ancienneté est l'abondance de ce récit au Brésil (I6 versions selon Bráulio do

I. Dans: <http://home.utad.pt/ letras/SiteLiteratura/>, fichier « Literatura da Tradição Oral », page 546 , notre traduction. 
Nascimento, auteur du catalogue du conte brésilien) et une version au Cap-Vert. De noter que cet archipel fut, au temps de l'empire portugais d'Outremer, un lieu de passage pour la traversée atlantique pour le Brésil. Est possible, donc, que cette histoire a eu traversé l'océan à cette période de communications intenses, et làbas a pris des fortes racines et grandit en popularité jusqu'à aujourd'hui.

Au niveau des contenus, on a déjà vu qu'il s'agît d'une histoire de friponnerie avec un motif central de nature scatologique, précisément la pointe de l'histoire que déclenche le rire. Dans une version espagnole recueillie à Segovia par Aurelio Espinosa fils, l'informant de l'histoire, un paysan de 40 ans, pousse encore plus loin ce motif: quand la victime, un curé de campagne, va attraper « l'oiseau », il s'aperçoit «d'une chose étrange ». Rapidement il retire la main, mais en faisant ça, la main frappe une pierre et, pour apaiser la douleur, le curé suce les doigts. Avec de la merde, bien sûr. (Espinosa Hijo II, I988, conte nº 32I « ¡Quieto, canario! »).

Il faut dire que dans le monde de langue espagnole, ce conte-type est aussi plus connu en Amérique du Sud qu'en Espagne (voir Camarena I991: 279-80).

Dans ce que concerne la forme, elle est toujours courte, bien que des fois l'histoire soit enchainée avec des contes-type du Géant Dupé (Fontes I975, conte no $36 \mathrm{~A}$ ) ou d'autres facéties (Alcoforado-Albán 200I, conte no 78). Néanmoins, la plupart des versions sont autonomes et racontés comme anecdotes. Il y a de nos jours une multitude d'adaptations de cette histoire aux arts du spectacle au Brésil: Marionnettes, théâtre, cinéma, conteurs urbains d'histoires, etc. Il ne faut qu'accéder au You-tube et chercher Pedro Malasartes, pour confirmer l'énorme popularité de ce trickster de l'autre côté de l'océan Atlantique. De plus, on peut trouver les aventures de ce héros malin sur des feuilletons de colportage en vers (nommés simplement cordel), très populaires au Brésil.

Un autre genre de récits courts, que nous avons au Portugal, aussi très populaires, sont les anecdotes sur les habitants d'une région du sud du pays: l'Alentejo. Le « compère alentejano » est le sujet de nombreuses anecdotes qui jouent sur le caractère paresseux et provincial de ces personnages. Je veux seulement faire noter que, dans une volte-face inouie, ces plaisanteries nous montrent aussi un alentejano malin, comparable, en gros, aux tricheurs que nous sommes en train d'aborder.

La figure du trickster n'apparaît pas sur n'importe quel genre narratif de la littérature de tradition orale. Au contraire, c'est exclusivement un personnage appartenant aux narratives réalistes. Et c'est dans cet environnement qu'il fait la critique des mœurs de la société ou bien des vices et vertus de la nature humaine.

Les contes du géant dupé servent surtout à dénoncer les inégalités sociales, même si le dispositif du récit renverse la situation réelle: dans la fiction le personnage le plus faible est toujours le vainqueur.

Les facéties sont le royaume des benêts, qui s'agroupent deux par deux: mèrefils ; mari-femme ; mari-amant ; prêtre-sacristain, etc. Le trickster apparaît comme un troisième élément (solitaire) qui va détruite l'harmonie asymétrique entre ces personnages, et ou bien qu'il va se profiter de cette situation pour être adjuvant d'un côté, ou bien qu'il agît en profit personnele. La nature astucieuse de ce héros est néanmoins relative, car c'est toujours le personnage le plus pauvre et le moins âgé le plus doué de cervelle. Étant donné ça, on voit pourquoi est si facile aux classes plus basses de la société à se voir réfléchies dans les comportements 
et désirs primaires de ces personnages. Ces faits impliquent un désir inconscient d'accéder à des biens, que, dans la vie réelle, leur sont inaccessibles, ou presque.

Finalement, les plaisanteries sur le «voisin stupide » sont des outils à double visage, qui, dans le domaine des identités, participent dans la construction (ou destruction) de stéréotypes. Dans le cas portugais, les alentejanos sont ciblés d'une image négative qu'ils prennent dans leurs mains et la changent, pour s'en sortir bien de la situation et renforcer sa propre identité régionale, avec un mode de vie positif et caractère malin.

Dans le domaine de la classification, ce genre de récits, vue comme un ensemble, présente quelques traits caractéristiques.

On peut faire une analogie avec les légendes et dire qu'une partie des récits facétieux sont « migratoires » car ils se trouvent dans plusieurs pays, et une autre partie de ces narratives sont « locales », une fois qu'ils se trouvent seulement dans les traditions orales nationales, voire, locaux. Au niveau de la classification de ces narratives, les premiers ont une place plus sûre sur les catalogues nationaux sous la catégorie de contes facétieux. Les derniers, restent assemblés surtout dans des collections d'anecdotes et plaisanteries. Est-ce-que la particularité d'être régionaux est suffisante pour éloigner ces derniers de la catégorie de " contes facétieux »? Quel est, ainsi, le limite du conte facétieux? Et de l'anecdote/plaisanterie? Les récits de trickster, que nous venons d'aborder, sont bien dans intersection entre l'un et l'autre, créant un espace commun entre les deux, une sorte de pont que permet « d'ouvrir » la catégorie anecdote et de la mettre, elle aussi, comme sujet d'étude des chercheurs du conte facétieux.

\section{Références bibliographiques}

Alcoforado, Doralice F. Xavier; Maria del Rosário SuÁrez Albán (200I): Contos Populares Brasileiros (Bahia). Recife: Fundação Joaquim Nabuco/Editora Massangana.

CAmarena, Julio (I99I): Cuentos Tradicionales de León. Vol. II. Madrid: Seminario Menéndez Pidal/Diputación Provincial de León.

DA MATTA, Roberto (I983): Carnavais, Malandros e Heróis. Rio de Janeiro: Zahar Editores [Chap. V: «Pedro Malasartes e os paradoxos da malandragem», p. 194-235].

Espinosa (Hijo), Aurélio M. (I988): Cuentos Populares de Castilla y León. Tomo II. Madrid: Consejo Superior de Investigaciones Científicas.

FonTES, Manuel Bráulio da Costa (1975): Portuguese Folktales from California. [Ph D Thesis]. Los Angeles: University of California.

Griaule, Marcel; Germaine Dieterlen (1965): Le Renard Pâle (Tome I, Fasc. I: Le mythe cosmogonique. La création du Monde). Paris: Institut d'Ethnologie.

RADIN, Paul (I972): The Trickster, a study in American Indian mythology. New York: Schocken Books.

Rodriguez Garcia, Isabel (I985): « Cuentos de Pedro Malasartes en el Brasil ». Revista de Dialectología y Tradiciones Populares Tomo XL (I985): 239-257.

Street, Brian V. (I972): «The Trickster Theme: Winebago and Azande». Dans André Singer; Brian STREet (eds.). Zande Themes, essays presented to Sir Edward Evans-Pritchard. New Jersey: Rowman and Littlefield, p. 82-IO4. 


\section{Images}

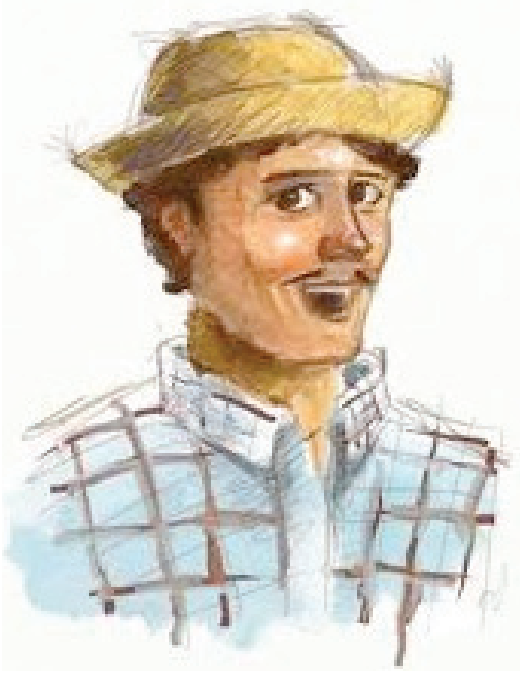

I. Pedro Malasartes

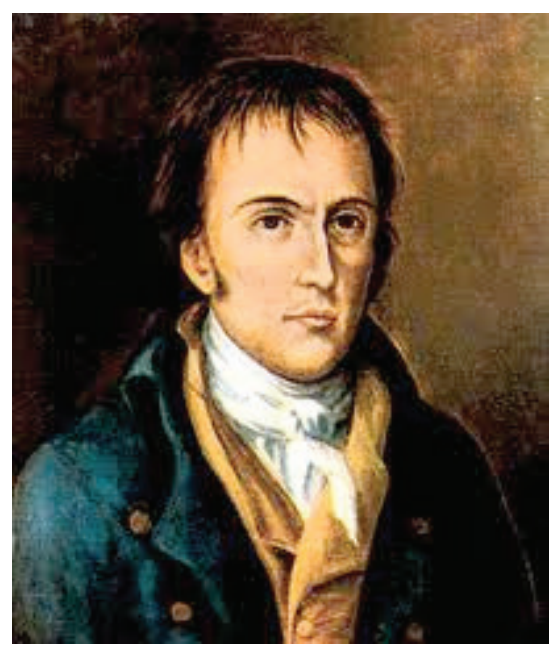

2. Manuel Maria Barbosa du Bocage

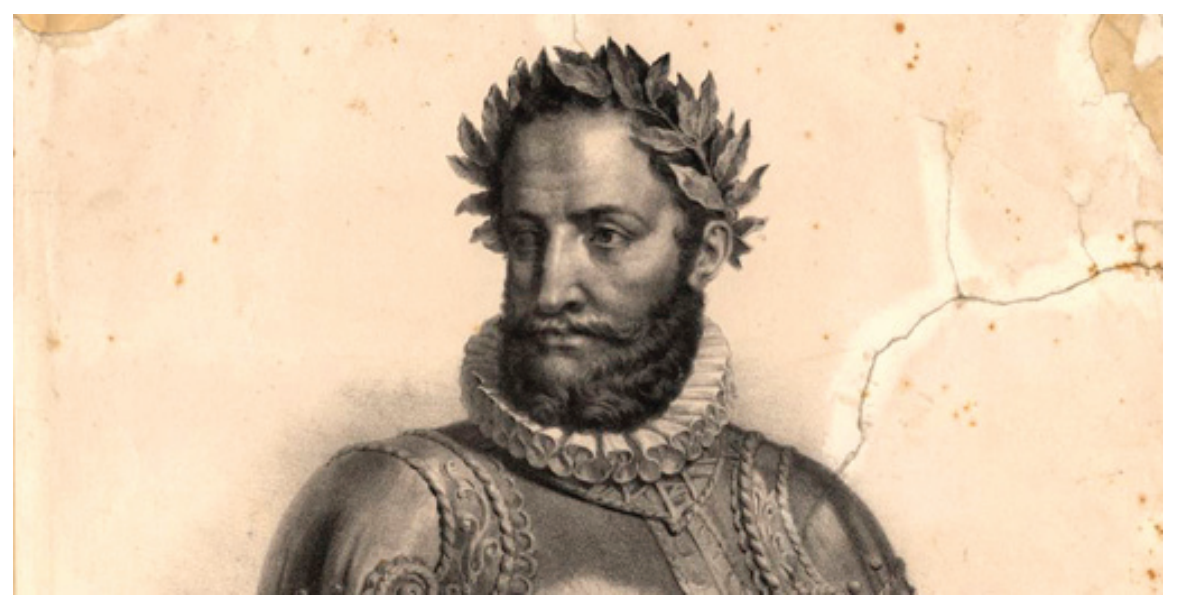

3. Luis Vaz de Camões 